\title{
DUMB-BELL GALAXIES IN SOUTHERN CLUSTERS: CATALOG AND PRELIMINARY STATISTICAL RESULTS
}

\author{
G. Vettolani ${ }^{1}$,L. Gregorini ${ }^{1,2}$, P. Parma ${ }^{1}$, H.R. de Ruiter ${ }^{3}$ \\ 1 Istituto di Radioastronomia del CNR, Bologna, Italy \\ 2 Dipartimento di Astronomia, Università di Bologna, Italy \\ 3 Osservatorio Astronomico, Bologna, Italy
}

\section{Introduction}

The dominant galaxy of a rich cluster is often an object whose formation and evolution is closely connected to the dynamics of the cluster itself. Hoessel (1980) and Schneider et al. (1983) estimate that $50 \%$ of the dominant galaxies are either of the dumb-bell type or have companions at projected distances less than $20 \mathrm{kpc}$, which is far in excess of the number expected from chance projection (see also Rood and Leir 1979).

Presently there is no complete sample of these objects, with the exception of the listing of dumb-bell galaxies in BM type I and I-II clusters in the Abell statistical sample of Rood and Leir (1979).

Recent dynamical studies of dumb-bell galaxies in clusters (Valentijn and Casertano, 1988) still suffer from inhomogeneity of the sample. The fact that it is a mixture of optically and radio selected objects may have introduced an unknown biases, for instance if the probability of radio emission is enhanced by the presence of close companions (Stocke, 1978, Heckman et al. 1985, Vettolani and Gregorini 1988) a bias could be present in their velocity distribution. However this situation is bound to improve: a new sample of Abell clusters in the Southern Hemisphere has been constructed (Abell et al., 1988 hereafter ACO), which has several advantages over the original northern catalog. The plate material (IIIaJ plates) is of better quality and reaches fainter magnitudes. This makes it possible to classify the cluster types with a higher degree of accuracy, as well as to fainter magnitudes. We have therefore decided to reconsider the whole problem constructing an new sample of dumb-bell galaxies homogeneously selected from the ACO survey.

\section{Selection rules and samples}

We have extracted from the ACO catalog two samples of clusters which were independently inspected on the ESO-SRC J plates by two of us (with subsamples inspected more than once at different times); the brightest member was classified according to the rules described below.

The first sample has been prepared in order to obtain a complete homogeneous volume limited sample of dumb-bell galaxies for further study of their dynamics, 
radio emission etc. It has been constructed examining all the BM type I an I-II clusters, independently of any other cluster parameter.

The second sample contains all clusters, regardless of their BM type, with distance either measured or estimated (Scaramella et al. 1989), less than $z=0.07$. This sample has been prepared in order to study the properties of dumb-bell galaxies in relation to the cluster environment (dominant/non-dominant cluster galaxy) and their space density, formation probability etc.

We classified the brightest member according to a five mark classification in which 4 denotes a dumb-bell galaxy, essentially according to the original definition given by Matthews et al. (1964): two objects with a difference in magnitude of at most 2 magnitudes, embedded in a common halo. In class 3 clusters there are two galaxies inside the halo, but with a large difference in luminosity (visually estimated as $\geq 2$ magnitudes). In classes 2 and 1 there is a companion galaxy outside the envelope either with a large luminosity difference (2) or with similar luminosity but at a distance from the brightest cluster galaxy which is more than twice the radius of the latter (1). These two classes could contain a few real dumb-bells, but are mainly composed of optical pairs. Many objects will simply be satellite galaxies. Only spectroscopy and surface photometry will help to disentangle these cases. In class 0 clusters the brightest member is fairly isolated from other cluster galaxies.

A catalog of clusters of type 3 and 4 for the volume limited sample is given in Table I.

\section{Selection effects}

These selection criteria are clearly subjective and rather strongly depend on one, or a combination, of the following parameters: distance, galactic latitude, cluster richness.

Distance The effect of distance is to increase the number of galaxies per unit area, thus increasing the number of apparently close ellipticals. Moreover common halos are no longer clearly discernable because of their limited angular extent.

Galactic latitude The effect of galactic latitude is twofold: on the one hand the increasing number of stars per unit area contaminates the sample of Galaxy/Star pairs misclassified as dumb-bells beyond the magnitude at which stars and galaxies can be easily separated visually (around 17-18 mags), on the other hand increasing absorption dims the halo to the point at which a true dumb-bell is misclassified as a pair of $\mathrm{E}$ galaxies because of the lack of a common halo.

Cluster Richness The probability of chance superposition of two E galaxies is clearly dependent on the cluster richness. Especially at large distances, when the envelope is not clearly visible, this effect increases the number of interlopers.

Evidently also the plate scale and the color have important effects, but all clusters were inspected on the same plate material (ESO SERC J Sky Atlas film copies) so in this respect the present search is homogeneous. 
Table I Dumb-bell galaxies in southern clusters $(\mathrm{z}<0.07)$

\begin{tabular}{|c|c|c|c|c|c|c|}
\hline Name & RA (1950) & DEC (1950) & M 10 & $\mathbf{R}$ & Class & BM type \\
\hline 419 & 30600 & -2353 & 16.0 & 0 & 3 & I-II \\
\hline 533 & 45912 & -2242 & 15.5 & 0 & 3 & III \\
\hline 548 & 54454 & -2539 & 14.6 & 2 & 4 & I-II \\
\hline 2401 & 215536 & -2021 & 15.6 & 1 & 3 & I \\
\hline 2412 & 220118 & -2141 & 16.1 & 0 & 4 & I-II \\
\hline 2799 & 03506 & -3924 & 16.0 & 1 & 3 & I-II \\
\hline 2800 & 03530 & -2522 & 15.6 & 1 & 4 & III \\
\hline 2819 & 04342 & -6352 & 15.8 & 2 & 3 & I-II \\
\hline 2824 & 04606 & -2137 & 15.3 & 0 & 4 & III \\
\hline 2854 & 05836 & -5048 & 15.6 & 1 & 3 & I-II \\
\hline 2860 & 10148 & -4003 & 16.0 & 0 & 4 & I \\
\hline 2911 & 12348 & -3814 & 16.1 & 1 & 4 & I-II \\
\hline 3009 & 22018 & -4848 & 16.1 & 1 & 3 & $\mathrm{I}$ \\
\hline 3089 & 30612 & -3654 & 15.6 & 0 & 3 & I-II \\
\hline 3093 & 30912 & -4735 & 16.2 & 2 & 3 & I \\
\hline 3094 & 30918 & -2707 & 16.1 & 2 & 3 & I-II \\
\hline 3095 & 31018 & -2720 & 16.1 & 0 & 3 & I-II \\
\hline 3104 & 31236 & -4536 & 16.0 & 0 & 3 & $I$ \\
\hline 3111 & 31606 & -4555 & 16.1 & 1 & 3 & I-II \\
\hline 3112 & $\begin{array}{ll}31612 \\
\end{array}$ & -4425 & 15.9 & 2 & 3 & I \\
\hline 3151 & 33824 & -2852 & 15.9 & 1 & 4 & I-II \\
\hline 3158 & 34142 & -5348 & 15.6 & 2 & 4 & I-II \\
\hline 3164 & 34442 & -5712 & 15.5 & 0 & 3 & I-II \\
\hline 3225 & 40824 & -5944 & 15.6 & 0 & 3 & II \\
\hline 3266 & 43030 & -6135 & 15.3 & 2 & 4 & I-II \\
\hline 3323 & 50924 & -2903 & 16.1 & 0 & 3 & I \\
\hline 3368 & 54824 & -2233 & 16.1 & 0 & 4 & I-II \\
\hline 3390 & 62318 & -3719 & 14.7 & 1 & 3 & II \\
\hline 3391 & 62512 & -5339 & 16.1 & 0 & 4 & I \\
\hline 3395 & 62630 & -5422 & 15.9 & 1 & 3 & II \\
\hline 3497 & 115730 & -3107 & 16.0 & 0 & 3 & I-II \\
\hline 3505 & 120606 & -3410 & 16.0 & 1 & 3 & I-II \\
\hline 3528 & 125136 & -2845 & 15.9 & 1 & 4 & II \\
\hline 3530 & 125254 & -3005 & 15.6 & 0 & 4 & I-II \\
\hline 3532 & 125436 & -3006 & 15.8 & 0 & 4 & II-III \\
\hline 3556 & 132118 & -3124 & 16.0 & 0 & 3 & I \\
\hline 3559 & 132706 & -2916 & 15.3 & 3 & 4 & I \\
\hline 3560 & 132900 & -3258 & 14.7 & 3 & 3 & I \\
\hline 3570 & $1343 \cdot 54$ & -3740 & 15.5 & 0 & 4 & I-II \\
\hline 3581 & 140436 & -2647 & 15.2 & 0 & 3 & I \\
\hline 3695 & 203136 & -3600 & 16.1 & 2 & 4 & I \\
\hline 3716 & 204754 & -5254 & 14.9 & 1 & 4 & I-II \\
\hline 3744 & 210418 & -2541 & 14.5 & 1 & 4 & II-III \\
\hline 3771 & 212606 & -5102 & 16.1 & 0 & 4 & III \\
\hline 3822 & 215036 & -5805 & 16.2 & 2 & 4 & II-III \\
\hline 3880 & 222500 & -3050 & 15.6 & 0 & 3 & II \\
\hline
\end{tabular}




\section{Dumb-bells as brightest cluster members}

Bautz-Morgan (Bautz and Morgan 1970,hereafter BM) I and I-II clusters of galaxies are dominated by an outstandingly large, luminous galaxy, almost invariably a cD, as defined by Mattews et al. (1964), while at the opposite extreme in BM class III clusters no member stands out against the other galaxies. Rood and Leir (1979), examining the Abell (1958) statistical sample (which is confined to clusters of richness 1 or greater) showed that 23 per cent (28 out of 112) of the dominant galaxies in BM I and I-II clusters are binary supergiant galaxies. On the contrary BM III type clusters contain only 3 per cent of multiple galaxies. This implies that the $\mathrm{cD}$ dominated clusters are physically different from the BM III clusters as to the multiple nature of the dominant galaxies.

The simple statistics in Table II on the present complete volume limited sample confirms the Rood and Leir (1979) results on the northern clusters sample, namely that in the clusters dominated by an outstanding bright galaxy $(\mathrm{cD})$ the probability of having a dumb-bell (or a multiple component supergiant galaxy) is 2.5 times higher than in other BM type clusters.

\section{References}

Abell,G.O. 1958 Astrophys. J. Suppl. 3, 211

Abell,G.O., Corwin,H., Olowin,R. 1989 Astrophys. J. Suppl. 70, 1

Bautz,L.P., Morgan,W.W. 1970 Astrophys. J. Lett. 162, L149

Heckman,,T.M. et al. 1985 Astrophys. J. 288, 122

Hoessel et al. 1980, Astrophys. J. 241, 486

Matthews,T.A., Morgan,W.W., Schmidt,M. 1964 Astrophys. J. 140, 35

Rood,H.J., Leir,A.W. 1979 Astrophys. J. Lett. 231, L3

Schneider et al. 1983, Astrophys. J. 264, 337

Stocke 1978, Astron. J. 83, 348

Valentijn and Casertano 1988, Astron. Astrophys. 206, 27

Vettolani,G., Gregorini, L. 1988 Astron. Astrophys. 189, 39

Table II Clusters with $\mathrm{z}<\mathbf{0 . 0 7}$

\begin{tabular}{ccc} 
Multiplicity & I and I-II & Fm II to III \\
\hline all & 81 & 76 \\
$0-2$ & $47(58 \%)$ & $64(84 \%)$ \\
3 and 4 & $34(42 \%)$ & $12(16 \%)$ \\
\hline
\end{tabular}

